\title{
A theoretical approach to the interaction between buckling and resonance instabilities
}

\author{
Alberto Carpinteri*, Marco Paggi \\ Politecnico di Torino, Department of Structural and Geotechnical Engineering, \\ Corso Duca degli Abruzzi 24, 10129 Torino, Italy
}

\begin{abstract}
The paper deals with the interaction between buckling and resonance instabilities of mechanical systems. Taking into account the effect of geometric nonlinearity in the equations of motion through the geometric stiffness matrix, the problem is reduced to a generalized eigenproblem where both the loading multiplier and the natural frequency of the system are unknown. According to this approach, all the forms of instabilities intermediate between those of pure buckling and pure forced resonance can be investigated. Numerous examples are analyzed, including: discrete mechanical systems with one to $n$ degrees of freedom, continuous mechanical systems such as oscillating deflected beams subjected to a compressive axial load, as well as oscillating beams subjected to lateral-torsional buckling. A general finite element procedure is also outlined, with the possibility to apply the proposed approach to any general bi- or tri-dimensional framed structure. The proposed results provide a new insight in the interpretation of coupled phenomena such as flutter instability of long-span or high-rise structures.
\end{abstract}

Key words: Buckling; Resonance; Flutter; Discrete systems; Continuous systems; Finite elements.

\section{Introduction}

Buckling, resonance and flutter are the main forms of instability of the elastic equilibrium of structural systems. In buckling instability, by removing the hypothesis of small displacements so that the deformed structural configuration can be distinguished from the undeformed one, it is possible to show that the

\footnotetext{
* Corresponding author. Tel. +39-011-564-4850 Fax +39-011-564-4899

Email address: alberto. carpinteri@polito.it (Alberto Carpinteri).
} 
solution of an elastic problem can represent a condition of stable, neutral or unstable equilibrium, depending on the magnitude of the applied load. As is well-known, buckling is usually observed in slender structural elements subjected to a compressive stress field, such as columns of buildings, machine shafts, struts of trusses, thin arches and shells. In some cases, elastic instability can also take place for special loading and geometrical conditions, as for example in the lateral torsional buckling of slender beams [1].

The phenomenon of resonance is also particularly important in structural engineering. It represents a form of dynamic instability, which occurs when an external periodic frequency matches one of the natural frequencies of vibration of the mechanical system. In this case, therefore, structural design deals with the determination of such dangerous natural frequencies according to modal analysis [2-4].

Finally, the phenomenon of flutter is a form of aeroelastic instability observed in long-span or high-rise structures subjected to wind loads, such as towers, tall buildings [5] and suspended [6-8] or cable-stayed bridges [9]. In this case, the instability is attributed to motion-induced or self-excited forces, which are loads induced or influenced by the deformation of the structure itself $[10,11]$. Forces originated in this way modify the initial deformation of the mechanical system which, consequently, leads to modified forces, and so on. This feed-back mechanism can give rise to an amplification effect on the initial deformation, leading to premature failure of the structure. The well-known dramatic Tacoma Narrows bridge disaster of 1940 is a famous example of this catastrophic interaction, and it is still very much in the public eye today. In this field, which is not at present completely understood, aeroelastic instability is often considered as the result of the interaction between buckling (static) and resonance (dynamic) instabilities. However, only a few theoretical formulations have been proposed for modelling aerodynamic forces and, in most investigations, empirical models are set up in which the parameters related to the fluid-structure interaction are established by experiments [12].

In the present contribution, we deal with the phenomenon of interaction between buckling and resonance instabilities. A state-of-the-art survey of the existing Literature shows that this problem has been mainly addressed in the field of multi-parameter stability theory [13-20], where the conditions for stability of a mechanical system are studied with reference to a perturbation of the problem parameters. In this framework, instability domains of some continuous oscillatory systems subjected to buckling loads were provided $[17,18]$. However, to make the problem analytically treatable, the analysis was mainly limited to certain continuous mechanical systems such as deflected beams and beams experiencing lateral torsional buckling. More importantly, the concept of geometric stiffness matrix, typical of structural engineering approaches, was totally neglected. 
On the other hand, in the field of bridge engineering, the influence of the geometric nonlinearity is usually taken into account by including the contribution of the self-induced aerodynamic forces to the applied loads (see e.g. [9,21,22] and [23] for a detailed overview of the mathematical methods). Such nonconservative aerodynamic forces are put in relationship with the displacements and the velocities of the points of the mechanical system according to the socalled flutter derivatives that have to be experimentally determined in the laboratories [24]. In these approaches, the onset of flutter instability corresponds to the condition of vanishing structural dumping. Therefore, structural damping seems to play a fundamental role, although it is practically impossible to be analytically evaluated but only experimentally estimated [21].

From the mathematical point of view, it is important to note that pure buckling, pure resonance, and also flutter instabilities are usually mathematically treated as eigenvalue problems. In this paper, we propose a mathematical theory for the analysis of buckling and resonance interaction, with the possibility to give an insight onto the mechanisms leading to flutter instability. In the mathematical treatment, a special focus will be given to the role played by the geometric stiffness matrix, which contributes to the reduction of the global elastic stiffness due to the effect of the geometric nonlinearity. This represents a novelty of our approach with respect to the models available in the Literature. As it will be shown in the sequel, the use of the geometric stiffness matrix may provide the proper link between multi-parameter stability theory, typical of rational mechanics, and the bridge engineering approach, typical of bridge engineers.

According to this formulation, we will demonstrate that the interaction between buckling and resonance leads to a generalized eigenvalue problem where both the buckling loads and the natural frequencies of the system are unknown and represent the eigenvalues. This approach will permit to inspect all the forms of structural instability intermediate between pure buckling and pure resonance. These limit cases are instead observed either when the dynamics of the system is neglected, or when the external buckling forces are equal to zero.

The effectiveness of the proposed methodology will be demonstrated with respect to not only discrete mechanical systems with one to $n$ degrees of freedom, but also for continuous mechanical systems such as oscillating deflected beams and beams showing lateral-torsional coupled deformations. Finally, a general procedure is established in the framework of the finite element method. 


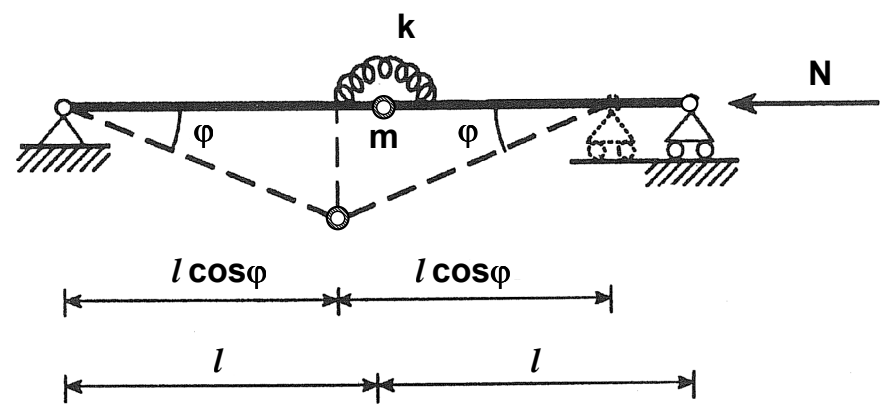

Figure 1. Scheme of the first one-degree of freedom system analyzed.

\section{Discrete mechanical systems}

\subsection{Discrete mechanical systems with one degree of freedom}

Let us consider the mechanical system shown in Fig.1, consisting of two rigid rods connected by an elastic hinge of rotational rigidity $k$ and constrained at one end by a hinge and at the other by a roller support. A mass $m$ is placed in correspondence of the intermediate elastic hinge and the system is loaded by a horizontal axial force $N$. Considering the absolute rotation $\varphi$ of the two arms as the generalized coordinate, the total potential energy, $W$, and the kinetic energy, $T$, of the whole system are:

$$
\begin{aligned}
W(\varphi) & =\frac{1}{2} k(2 \varphi)^{2}-2 N l(1-\cos \varphi) \\
T(\dot{\varphi}) & =\frac{1}{2} m\left[\frac{\mathrm{d}}{\mathrm{d} t}(l \sin \varphi)\right]^{2}+\frac{1}{2} m\left[\frac{\mathrm{d}}{\mathrm{d} t}(l-l \cos \varphi)\right]^{2}=\frac{1}{2} m l^{2} \dot{\varphi}^{2} .
\end{aligned}
$$

The equation of motion can be determined by writing the Lagrange's equation:

$$
\frac{\partial}{\partial t}\left(\frac{\partial T}{\partial \dot{\varphi}}\right)-\frac{\partial T}{\partial \varphi}=-\frac{\partial W}{\partial \varphi}
$$

In the present case, this yields:

$$
m l^{2} \ddot{\varphi}=-4 k \varphi+2 N l \sin \varphi
$$

which can be suitably linearized in correspondence of $\varphi=0$ :

$$
m l^{2} \ddot{\varphi}=-4 k \varphi+2 N l \varphi
$$

Looking for the solution to Eq.(4) in the general form $\varphi=\varphi_{0} \mathrm{e}^{\mathrm{i} \omega t}$, where $\omega$ denotes the natural angular frequency of the system, we obtain the following 


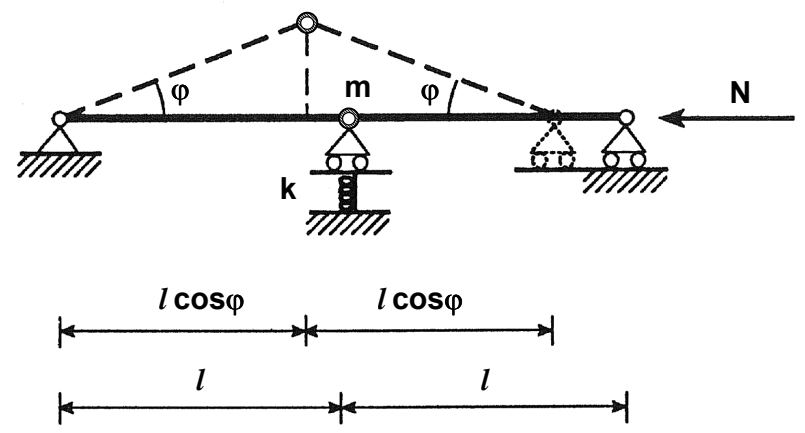

Figure 2. Scheme of the second one-degree of freedom system analyzed.

equation which provides the conditions of equilibrium of the system:

$$
\left(4 k-2 N l-\omega^{2} m l^{2}\right) \varphi_{0}=0 .
$$

A nontrivial solution to Eq.(5) exists if and only if the term in brackets is equal to zero. This critical condition corresponding to the bifurcation of the equilibrium establishes a one-to-one relationship between the applied axial force, $N$, and the angular frequency, $\omega$ :

$$
N=\frac{2 k}{l}-\frac{m l}{2} \omega^{2}
$$

Moreover, Eq.(6) admits two important limit conditions for, respectively, $N=$ 0 and $m=0$. In the former case, Eq.(6) gives the natural angular frequency of the system according to pure modal analysis, that is $\omega_{1}=\sqrt{4 k / m l^{2}}$. In the latter, the pure critical Eulerian load is obtained, that is $N_{1}=2 k / l$. Dividing Eq.(6) by $N_{1}$, we obtain the following relationship between $N$ and $\omega$ in a nondimensional form:

$$
\left(\frac{\omega}{\omega_{1}}\right)^{2}+\left(\frac{N}{N_{1}}\right)=1
$$

As a second example, let us consider the mechanical system shown in Fig.2, consisting in two rigid rods on three supports, of which the intermediate one is assumed to be elastically compliant with rigidity $k$. As in the previous case, a mass $m$ is placed in correspondence of the intermediate hinge and the system is loaded by a horizontal axial force $N$. Considering the absolute rotation $\varphi$ of the two arms as the generalized coordinate, the total potential energy, $W$, and the kinetic energy, $T$, of the whole system are:

$$
\begin{aligned}
W(\varphi) & =\frac{1}{2} k(l \sin \varphi)^{2}-2 N l(1-\cos \varphi), \\
T(\dot{\varphi}) & =\frac{1}{2} m l^{2} \dot{\varphi}^{2} .
\end{aligned}
$$

Following the procedure discussed above, we determine the equation of motion 
by employing the Lagrange's equation (2):

$$
m l^{2} \ddot{\varphi}=-l \sin \varphi(k l \cos \varphi-2 N)
$$

which can be suitably linearized in correspondence of $\varphi=0$ :

$$
m l^{2} \ddot{\varphi}=-l \varphi(k l-2 N)
$$

Looking for the solution to Eq.(10) in the general form $\varphi=\varphi_{0} \mathrm{e}^{\mathrm{i} \omega t}$, where $\omega$ denotes the natural angular frequency of the system, we obtain the following condition of equilibrium of the system:

$$
\left(k l^{2}-2 N l-\omega^{2} m l^{2}\right) \varphi_{0}=0 .
$$

As in the previous example, by setting equal to zero the term in brackets, we obtain a one-to-one relationship between the applied axial force, $N$, and the angular frequency, $\omega$ :

$$
N=\frac{k l}{2}-\frac{m l}{2} \omega^{2}
$$

This equation admits two important limit conditions for, respectively, $N=0$ and $m=0$. In the former case, Eq.(12) gives the natural angular frequency of the system according to pure modal analysis, that is $\omega_{1}=\sqrt{k / m}$. In the latter, the pure critical Eulerian load for buckling instability is obtained, that is $N_{1}=k l / 2$. Dividing Eq.(12) by $N_{1}$, we obtain the same relationship between the nondimensional terms $N / N_{1}$ and $\left(\omega / \omega_{1}\right)^{2}$ as in the previous example (see Eq.(7)).

A graphical representation of the condition (7) in Fig.3 shows that the resonance frequency is a decreasing function of the compressive axial load. This demonstrates, for the mechanical systems with a single degree of freedom, that the condition of bifurcation of the equilibrium can be reached for a compressive axial force, $N$, lower than the Eulerian buckling load, $N_{1}$, provided that the system is subjected to an external excitation with frequency $\omega$ given by Eq.(7). Conversely, failure due to resonance can take place for $\omega<\omega_{1}$, provided that the system is loaded by an axial force $N$ given by Eq.(7).

Finally, the issue of stability or instability of the mechanical system in the correspondence of the bifurcation point can be discussed as in the static case, i.e. by evaluating the higher order derivatives of the total potential energy $W$. 


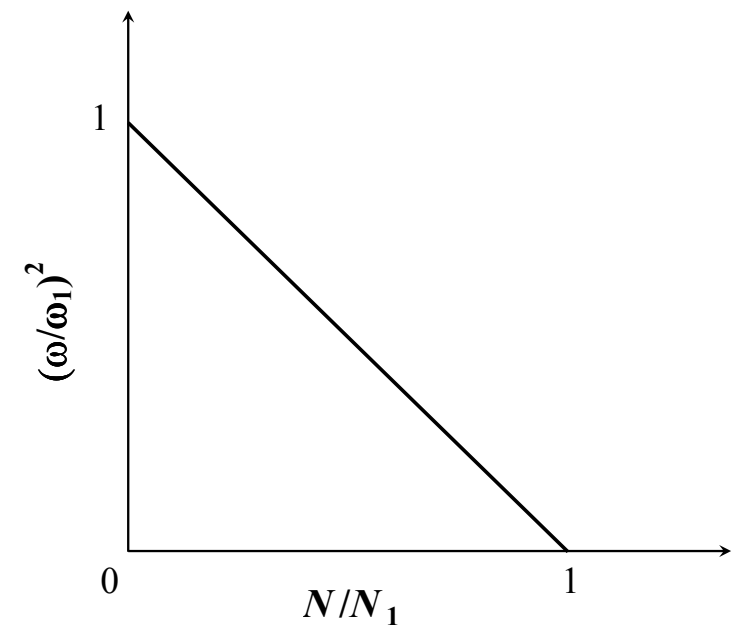

Figure 3. Nondimensional frequency vs. nondimensional axial force for the single degree of freedom systems.

\subsection{Discrete mechanical systems with $n$ degrees of freedom}

Let us consider the mechanical system with two degrees of freedom shown in Fig.4, consisting of three rigid rods connected by two elastic hinges of rotational rigidity $k$, and constrained at one end by a hinge and at the other by a roller support. A mass $m$ is placed in correspondence of the intermediate elastic hinges and the system is loaded by a horizontal axial force $N$. Assuming the vertical displacements $x_{1}$ and $x_{2}$ of the elastic hinges as the generalized coordinates, the total potential energy, $W$, and the kinetic energy, $T$, of the whole system are given by:

$$
\begin{aligned}
W\left(x_{1}, x_{2}\right)= & \frac{1}{2} k\left[\left(\arcsin \frac{x_{1}}{l}-\arcsin \frac{x_{2}-x_{1}}{l}\right)^{2}\right. \\
& \left.+\left(\arcsin \frac{x_{2}}{l}+\arcsin \frac{x_{2}-x_{1}}{l}\right)^{2}\right] \\
& -N l\left[3-\cos \left(\arcsin \frac{x_{1}}{l}\right)-\cos \left(\arcsin \frac{x_{2}}{l}\right)\right. \\
& \left.-\cos \left(\arcsin \frac{x_{2}-x_{1}}{l}\right)\right], \\
T\left(\dot{x_{1}}, \dot{x_{2}}\right)= & \frac{1}{2} m \dot{x_{1}}{ }^{2}+\frac{1}{2} m \dot{x_{1}}{ }^{2} x_{1}^{2}+\frac{1}{2} m \dot{x_{2}} \\
& +\frac{1}{2} m\left(\frac{2 x_{1} \dot{x_{1}}}{l}+\frac{x_{2} \dot{x_{2}}}{l}-\frac{x_{2} \dot{x_{1}}}{l}-\frac{x_{1} \dot{x_{2}}}{l}\right)^{2} .
\end{aligned}
$$

Performing a Taylor series expansion of Eq.(13) about the origin, and assuming 


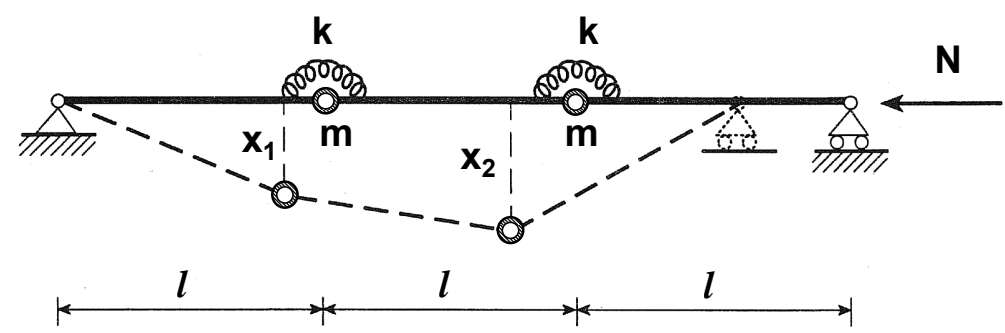

Figure 4. Scheme of the two-degrees of freedom system analyzed.

$x_{1} / l<1 / 10$ and $x_{2} / l<1 / 10$, we obtain:

$$
\begin{aligned}
& W\left(x_{1}, x_{2}\right) \cong \frac{k}{2 l^{2}}\left(5 x_{1}^{2}+5 x_{2}^{2}-8 x_{1} x_{2}\right)-\frac{N}{l}\left(x_{1}^{2}+x_{2}^{2}-x_{1} x_{2}\right), \\
& T\left(\dot{x_{1}}, \dot{x_{2}}\right) \cong \frac{1}{2} m{\dot{x_{1}}}^{2}+\frac{1}{2} m{\dot{x_{2}}}^{2} .
\end{aligned}
$$

The equations of motion are identified by considering the Lagrange's equations:

$$
\frac{\partial}{\partial t}\left(\frac{\partial T}{\partial \dot{x}_{i}}\right)-\frac{\partial T}{\partial x_{i}}=-\frac{\partial W}{\partial x_{i}}, \quad i=1,2 .
$$

In matrix form, they are:

$$
\left[\begin{array}{cc}
m & 0 \\
0 & m
\end{array}\right]\left\{\begin{array}{l}
\ddot{x}_{1} \\
\ddot{x}_{2}
\end{array}\right\}+\left[\begin{array}{cc}
\frac{5 k}{l^{2}} & -\frac{4 k}{l^{2}} \\
-\frac{4 k}{l^{2}} & \frac{5 k}{l^{2}}
\end{array}\right]\left\{\begin{array}{l}
x_{1} \\
x_{2}
\end{array}\right\}-N\left[\begin{array}{cc}
\frac{2}{l} & -\frac{1}{l} \\
-\frac{1}{l} & \frac{2}{l}
\end{array}\right]\left\{\begin{array}{l}
x_{1} \\
x_{2}
\end{array}\right\}=\left\{\begin{array}{l}
0 \\
0
\end{array}\right\}
$$

Looking for the solution to Eq.(16) in the general form $\{q\}=\left\{q_{0}\right\} \mathrm{e}^{\mathrm{i} \omega t}$, where $\omega$ denotes the natural angular frequency of the system, we obtain the following equation, written in symbolic form:

$$
\left(-\omega^{2}[M]+[K]-N\left[K_{g}\right]\right)\left\{q_{0}\right\}=\{0\}
$$

where $[M],[K]$ and $\left[K_{G}\right]$ denote, respectively, the mass matrix, the elastic stiffness matrix and the geometric stiffness matrix of the mechanical system. Their expressions can be simply obtained by comparying Eq.(17) with Eq.(16).

A nontrivial solution to Eq.(17) exists if and only if the determinant of the resultant coefficient matrix of the vector $\left\{q_{0}\right\}$ vanishes. This yields the following generalized eigenvalue problem:

$$
\operatorname{det}\left([K]-N\left[K_{g}\right]-\omega^{2}[M]\right)=0,
$$

where $N$ and $\omega^{2}$ represent the eigenvalues. For this example, Eq.(18) provides 
the following relationships between the eigenvalues $\omega$ and $N$ :

$$
\begin{aligned}
\omega^{2} & =\frac{k}{m l^{2}}-\frac{N}{m l}, \\
\omega^{2} & =3 \frac{k}{m l^{2}}-\frac{3 N}{m l} .
\end{aligned}
$$

As limit cases, if $m=0$, then we obtain the Eulerian buckling loads:

$$
\begin{aligned}
& N_{1}=\frac{k}{l}, \\
& N_{2}=\frac{3 k}{l},
\end{aligned}
$$

whereas, if $N=0$, we obtain the natural frequencies of the system:

$$
\begin{aligned}
\omega_{1} & =\sqrt{\frac{k}{m l^{2}}} \\
\omega_{2} & =\sqrt{\frac{9 k}{m l^{2}}} .
\end{aligned}
$$

As far as the eigenvectors are concerned, the system (17) yields the eigenvectors corresponding, respectively, to the eigenfrequencies (19a) and (19b) as functions of $N$ :

$$
\begin{aligned}
& x_{1}=\frac{4 k / l-N}{6 k / l-3 N} x_{2}, \\
& x_{1}=\frac{4 k / l-N}{14 k / l-5 N} x_{2} .
\end{aligned}
$$

Dividing Eqs.(19a) and (19b) by $\omega_{1}^{2}$, we derive the following nondimensional relationships between the eigenvalues:

$$
\begin{aligned}
& \left(\frac{\omega}{\omega_{1}}\right)^{2}=1-\left(\frac{N}{N_{1}}\right), \\
& \left(\frac{\omega}{\omega_{1}}\right)^{2}=\left(\frac{\omega_{2}}{\omega_{1}}\right)^{2}-\frac{N_{2}}{N_{1}}\left(\frac{N}{N_{1}}\right) .
\end{aligned}
$$

In analogy with the results for the single degree of freedom systems, a graphical representation of Eqs. (23a) and (23b) is provided in Fig.5. We notice that both the eigenfrequencies are decreasing functions of the compressive axial load. Starting from $N=0$, bifurcation of the equilibrium would correspond to pure resonance instability. Entering the diagram with a value of the nondimensional compressive axial force in the range $0<N / N_{1}<1$, the coordinates of the points of the two curves provide the critical eigenfrequencies of the mechanical system leading to bifurcation. Axial forces higher than $N_{1}$ in the 


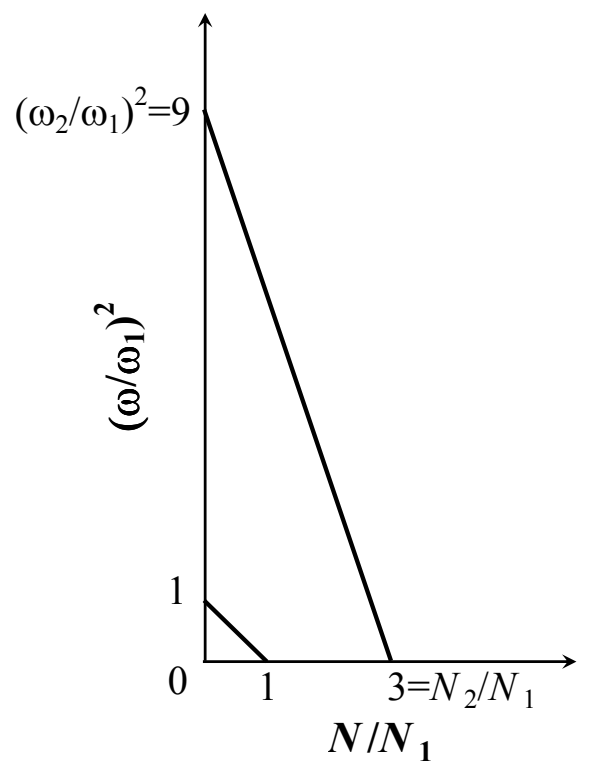

Figure 5. Nondimensional frequencies vs. nondimensional axial forces for the two-degrees of freedom system in Fig.4.

range $1<N / N_{1}<N_{2} / N_{1}$ can only be experienced if an additional constraint is introduced into the system. Moreover, we observe that the applied compressive load influences all the eigenfrequencies. In particular, for the present example, the influence of the axial load is greater on the highest frequency than on the lower one.

As a second example of a system with two degrees of freedom, let us examine that of Fig.6, which consists of three rigid rods on four supports, of which the central ones are assumed to be elastically compliant with rigidity $k$. A mass $m$ is placed in correspondence of the intermediate hinges and the system is loaded by a horizontal axial force $N$. Assuming the vertical displacements $x_{1}$ and $x_{2}$ of the elastic hinges as the generalized coordinates, the total potential energy, $W$, and the kinetic energy, $T$, of the whole system are given by $\left(x_{1} / l<1 / 10\right.$ and $\left.x_{2} / l<1 / 10\right)$ :

$$
\begin{aligned}
W\left(x_{1}, x_{2}\right)= & \frac{1}{2} k\left(x_{1}^{2}+x_{2}^{2}\right)-N l\left[3-\cos \left(\arcsin \frac{x_{1}}{l}\right)\right. \\
& \left.-\cos \left(\arcsin \frac{x_{2}}{l}\right)-\cos \left(\arcsin \frac{x_{2}-x_{1}}{l}\right)\right] \\
\cong & \frac{1}{2} k\left(x_{1}^{2}+x_{2}^{2}\right)-\frac{N}{l}\left(x_{1}^{2}+x_{2}^{2}-x_{1} x_{2}\right), \\
T\left(\dot{x_{1}}, \dot{x_{2}}\right) \cong & \frac{1}{2} m{\dot{x_{1}}}^{2}+\frac{1}{2} m{\dot{x_{1}}}^{2} .
\end{aligned}
$$




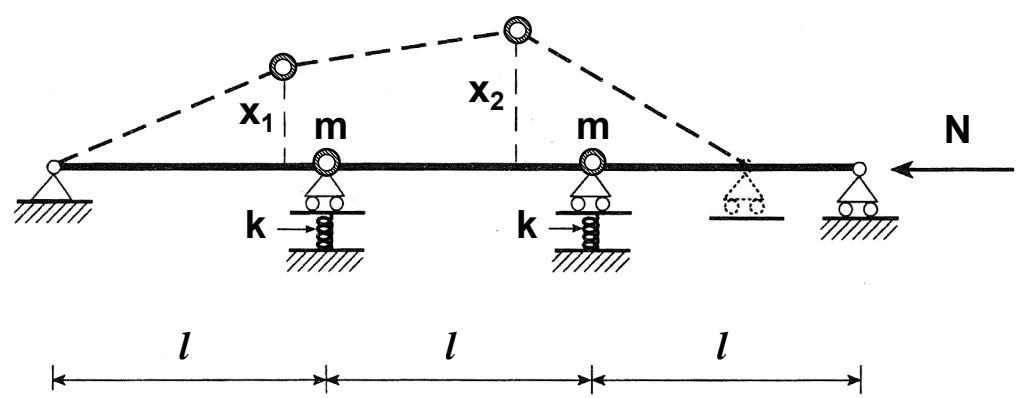

Figure 6. Scheme of the second two-degrees of freedom system analyzed.

In this case, the Lagrange's equations (15) yield the following matrix form:

$$
\left[\begin{array}{cc}
m & 0 \\
0 & m
\end{array}\right]\left\{\begin{array}{l}
\ddot{x}_{1} \\
\ddot{x}_{2}
\end{array}\right\}+\left[\begin{array}{ll}
k & 0 \\
0 & k
\end{array}\right]\left\{\begin{array}{l}
x_{1} \\
x_{2}
\end{array}\right\}-N\left[\begin{array}{cc}
\frac{2}{l} & -\frac{1}{l} \\
-\frac{1}{l} & \frac{2}{l}
\end{array}\right]\left\{\begin{array}{l}
x_{1} \\
x_{2}
\end{array}\right\}=\left\{\begin{array}{l}
0 \\
0
\end{array}\right\} .
$$

Looking for the solution to Eq.(25) in the general form $\{q\}=\left\{q_{0}\right\} \mathrm{e}^{\mathrm{i} \omega t}$, where $\omega$ denotes the natural angular frequency of the system, we obtain the following equation, written in symbolic form:

$$
\left(-\omega^{2}[M]+[K]-N\left[K_{g}\right]\right)\left\{q_{0}\right\}=\{0\},
$$

where $[M],[K]$ and $\left[K_{g}\right]$ denote, respectively, the mass matrix, the elastic stiffness matrix and the geometric stiffness matrix of the mechanical system. As it can be readily seen, the geometric stiffness matrix for this problem is the same as that of the previous example.

A nontrivial solution to Eq.(26) exists if and only if the determinant of the resultant coefficient matrix of the vector $\left\{q_{0}\right\}$ is equal to zero. This yields the following generalized eigenvalue problem:

$$
\operatorname{det}\left([K]-N\left[K_{g}\right]-\omega^{2}[M]\right)=0,
$$

where $N$ and $\omega^{2}$ are the eigenvalues of the system. For this example, Eq.(27) provides the following relationships between the eigenvalues:

$$
\begin{aligned}
\omega^{2} & =\frac{k}{m}-3 \frac{N}{m l}, \\
\omega^{2} & =\frac{k}{m}-\frac{N}{m l} .
\end{aligned}
$$

As limit cases, if $m=0$, we obtain the Eulerian buckling loads:

$$
\begin{aligned}
N_{1} & =\frac{1}{3} k l, \\
N_{2} & =k l,
\end{aligned}
$$




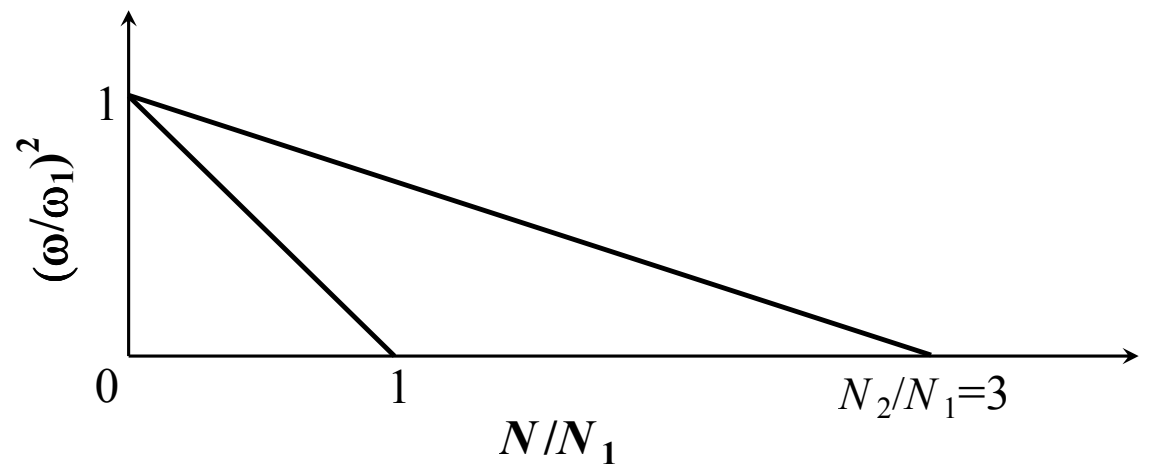

Figure 7. Nondimensional frequencies vs. nondimensional axial forces for the two-degrees of freedom system in Fig.6.

whereas, if $N=0$, then we obtain the natural frequencies of the system:

$$
\omega_{1}=\omega_{2}=\sqrt{\frac{k}{m}} .
$$

As far as the eigenvectors are concerned, the system (26) yields the eigenvectors corresponding, respectively, to the eigenfrequencies (28a) and (28b), as functions of the axial force, $N$ :

$$
\begin{aligned}
& x_{1}=\frac{N / l}{5 N / l-2 k} x_{2}, \\
& x_{1}=\frac{N / l}{3 N / l-2 k} x_{2} .
\end{aligned}
$$

Dividing Eqs.(28a) and (28b) by $\omega_{1}^{2}$, we obtain the following nondimensional relationships between the eigenvalues:

$$
\begin{aligned}
& \left(\frac{\omega}{\omega_{1}}\right)^{2}=1-\left(\frac{N}{N_{1}}\right), \\
& \left(\frac{\omega}{\omega_{1}}\right)^{2}=1-\frac{N_{1}}{N_{2}}\left(\frac{N}{N_{1}}\right) .
\end{aligned}
$$

A graphical representation of Eqs.(32a) and (32b) is provided in Fig.7. Also in this case, both the frequencies are decreasing functions of the compressive axial load. However, for the present example, the influence of the axial load is greater on the lower frequency of the system than on the higher one. 

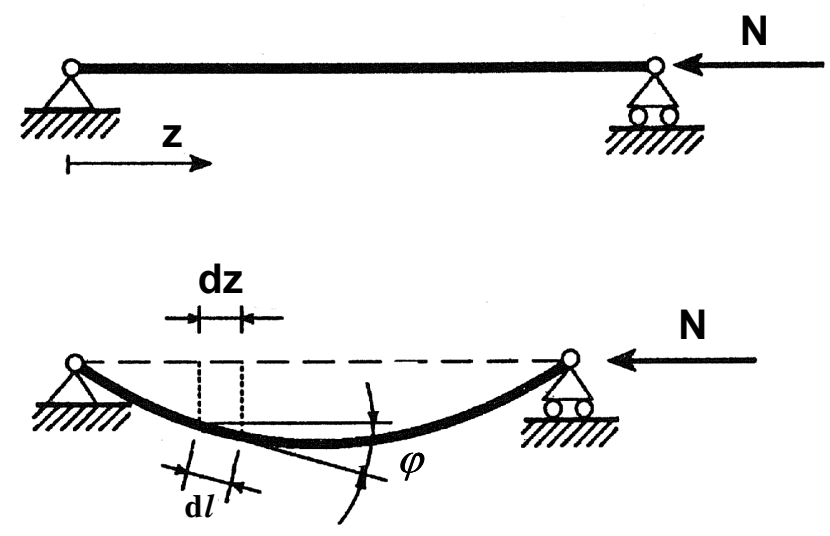

Figure 8. Undeformed and deformed configurations of a deflected beam under compressive axial force.

\section{Continuous mechanical systems}

\subsection{Oscillations of deflected beams under compressive axial loads}

Let us consider a slender elastic beam of constant cross-section, inextensible and not deformable in shear, though deformable in bending, constrained at one end by a hinge and at the other by a roller support, loaded by an axial force, $N$ (see Fig.8). In this case, with the purpose of analyzing the free flexural oscillations of the beam, the differential equation of the elastic line with second-order effects can be written by replacing the distributed load with the force of inertia:

$$
E I \frac{\partial^{4} v}{\partial z^{4}}+N \frac{\partial^{2} v}{\partial z^{2}}=-\mu \frac{\partial^{2} v}{\partial t^{2}}
$$

where $E I$ denotes the flexural rigidity of the beam and $\mu$ is its linear density (mass per unit length). Equation (33) can be rewritten in the following form:

$$
\frac{\partial^{4} v}{\partial z^{4}}+\beta^{2} \frac{\partial^{2} v}{\partial z^{2}}=-\frac{\mu}{E I} \frac{\partial^{2} v}{\partial t^{2}}
$$

where we have set $\beta^{2}=N / E I$.

Equation (34) is an equation with separable variables, the solution being represented as the product of two different functions, each one depending on a single variable:

$$
v(z, t)=\eta(z) f(t)
$$


Introducing Eq.(35) into Eq.(34), leads:

$$
\frac{\mathrm{d}^{4} \eta}{\mathrm{d} z^{4}} f+\beta^{2} \frac{\mathrm{d}^{2} \eta}{\mathrm{d} z^{2}} f+\frac{\mu}{E I} \eta \frac{\mathrm{d}^{2} f}{\mathrm{~d} t^{2}}=0
$$

Dividing Eq.(36) by the product $\eta f$, we find:

$$
-\frac{\frac{\mathrm{d}^{2} f}{\mathrm{~d} t^{2}}}{f}=\frac{\mu}{E I} \frac{\frac{\mathrm{d}^{4} \eta}{\mathrm{d} z^{4}}+\beta^{2} \frac{\mathrm{d}^{2} \eta}{\mathrm{d} z^{2}}}{\eta}=\omega^{2}
$$

where $\omega^{2}$ represents a positive constant, the left and the right hand-sides of Eq.(37) being at the most functions of the time $t$ and the coordinate $z$, respectively. From Eq.(37) there follow two ordinary differential equations:

$$
\begin{array}{r}
\frac{\mathrm{d}^{2} f}{\mathrm{~d} t^{2}}+\omega^{2} f=0 \\
\frac{\mathrm{d}^{4} \eta}{\mathrm{d} z^{4}}+\beta^{2} \frac{\mathrm{d}^{2} \eta}{\mathrm{d} z^{2}}-\alpha^{4} \eta
\end{array}
$$

with

$$
\alpha=\sqrt[4]{\frac{\mu \omega^{2}}{E I}}
$$

Whereas Eq.(38a) is the equation of the harmonic oscillator, with the wellknown complete integral

$$
f(t)=A \cos \omega t+B \sin \omega t
$$

Eq.(38b) has the following complete integral

$$
\eta(z)=C \mathrm{e}^{\lambda_{1} z}+D \mathrm{e}^{\lambda_{2} z}+E \mathrm{e}^{-\lambda_{1} z}+F \mathrm{e}^{-\lambda_{2} z}
$$

where $\lambda_{1}$ and $\lambda_{2}$ are functions of $\alpha$ and $\beta$ :

$$
\lambda_{1,2}=\sqrt{\frac{-\beta^{2} \pm \sqrt{\beta^{4}+4 \alpha^{4}}}{2}} .
$$

As in the modal analysis, the constants $A$ and $B$ can be determined on the basis of the initial conditions, while the constants $C, D, E$ and $F$ can be determined by imposing the boundary conditions. As it will be shown in the sequel, for a given value of $\beta$, the parameters $\omega$ and $\alpha$ can be determined by solving a generalized eigenvalue problem resulting from the imposition of the boundary conditions. From the mathematical point of view, this eigenvalue problem is analogous to that shown for the discrete systems. On the other hand, since we are considering a continuous mechanical system having infinite degrees of freedom, we shall obtain an infinite number of eigenvalues $\omega_{i}$ and 
$\alpha_{i}$, just as also an infinite number of eigenfunctions $f_{i}$ and $\eta_{i}$. The complete integral of the differential equation (33) may therefore be given the following form, according to the Principle of Superposition:

$$
v(z, t)=\sum_{i=1}^{\infty} \eta_{i}(z) f_{i}(t),
$$

with:

$$
\begin{aligned}
& f_{i}(t)=A_{i} \cos \omega_{i} t+B_{i} \sin \omega_{i} t \\
& \eta(z)=C_{i} \mathrm{e}^{\lambda_{1 i} z}+D_{i} \mathrm{e}^{\lambda_{2 i} z}+E_{i} \mathrm{e}^{-\lambda_{1 i} z}+F_{i} \mathrm{e}^{-\lambda_{2 i} z} .
\end{aligned}
$$

It is important to remark that the eigenfunctions $\eta_{i}$ are still orthonormal functions, as in the classical modal analysis (see the mathematical demonstration reported in the Appendix). This permits to determine the constants $A_{i}$ and $B_{i}$ in Eq.(44a) via the initial conditions (see also [1], Pag. 315):

$$
\begin{aligned}
v(z=0) & =v_{0}(z), \\
\frac{\partial v}{\partial t}(z=0) & =\dot{v}_{0}(z) .
\end{aligned}
$$

As regards the boundary conditions, let us consider as an example a beam supported at both ends, of length $l$ :

$$
\left\{\begin{array}{l}
\eta(0)=0, \\
\eta^{\prime \prime}(0)=0, \\
\eta(l)=0, \\
\eta^{\prime \prime}(l)=0,
\end{array} \Rightarrow\left[\begin{array}{cccc}
1 & 1 & 1 & 1 \\
\lambda_{1}^{2} & \lambda_{2}^{2} & \lambda_{1}^{2} & \lambda_{2}^{2} \\
\mathrm{e}^{\lambda_{1} l} & \mathrm{e}^{\lambda_{2} l} & \mathrm{e}^{-\lambda_{1} l} & \mathrm{e}^{-\lambda_{2} l} \\
\lambda_{1}^{2} \mathrm{e}^{\lambda_{1} l} & \lambda_{2}^{2} \mathrm{e}^{\lambda_{2} l} & \lambda_{1}^{2} \mathrm{e}^{-\lambda_{1} l} & \lambda_{2}^{2} \mathrm{e}^{-\lambda_{2} l}
\end{array}\right]\left\{\begin{array}{c}
C \\
D \\
E \\
F
\end{array}\right\}=\left\{\begin{array}{l}
0 \\
0 \\
0 \\
0
\end{array}\right\}\right.
$$

For a nontrivial solution to the system in Eq.(46), the determinant of the coefficient matrix has to vanish. The resulting eigenequation permits, for each given value of the parameter $\beta$, to determine the eigenvalues $\alpha_{i}$ of the system. Finally, the corresponding natural eigenfrequencies $\omega_{i}$ can be obtained by inverting Eq.(39).

As an illustrative example, the first three nondimensional frequencies of the simply supported beam shown in Fig.8 are reported in Fig.9 as functions of the applied nondimensional axial force. Parameters $\omega_{i}$ and $N_{i}$ denote, respectively, the $i$-th frequency of the system determined according to modal analysis and the $i$-th buckling load determined according to the Euler's formula. In close analogy with the discrete mechanical systems, the curves in the $\left(\omega / \omega_{1}\right)^{2}$ vs. $N / N_{1}$ plane are represented by straight lines. Also in this case, the coordinates of the points along these lines provide the critical conditions leading to the 


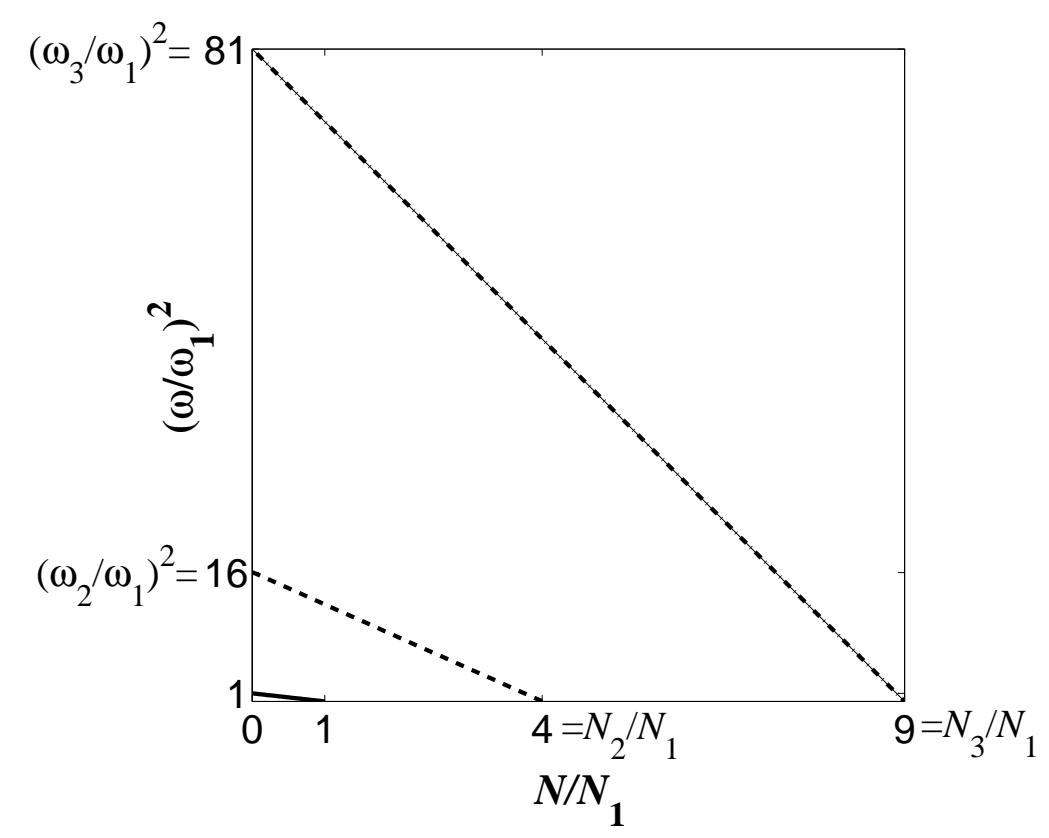

Figure 9. Nondimensional frequencies vs. nondimensional axial force for the continuous system in Fig.8.

system instability in terms of frequency of the excitation and magnitude of the applied compressive axial force.

\subsection{Oscillations and lateral-torsional buckling of beams}

Let us consider a beam of thin rectangular cross-section, constrained at the ends so that rotation about the longitudinal axis $Z$ is prevented. Let this beam be subjected to uniform bending by means of the application at the ends of two moments $m$ contained in the plane $Y Z$ of greater flexural rigidity (see Fig.10).

Considering a deformed configuration of the beam, with deflection thereof in the $X Z$ plane of smaller flexural rigidity, and simultaneous torsion about the $Z$ axis (see Fig.10), bending-torsional out-of-plane vibrations of the beam are described by the following partial differential equations:

$$
\begin{gathered}
E I_{y} \frac{\partial^{4} u}{\partial z^{4}}+m \frac{\partial^{2} \varphi_{z}}{\partial z^{2}}=-\mu \frac{\partial^{2} u}{\partial t^{2}} \\
-G I_{t} \frac{\partial^{2} \varphi_{z}}{\partial z^{2}}+m \frac{\partial^{2} u}{\partial z^{2}}=-\mu \rho^{2} \frac{\partial^{2} \varphi_{z}}{\partial t^{2}},
\end{gathered}
$$

where $u(z, t)$ and $\varphi_{z}(z, t)$ are, respectively, the out-of-plane deflection and the twist angle of the beam cross-section; $E I_{y}$ and $G I_{t}$ are the bending and torsional rigidities; $\mu$ is the mass of the beam per unit length, and $\rho=\sqrt{I_{P} / A}$ is the polar radius of inertia of the beam cross-section. 

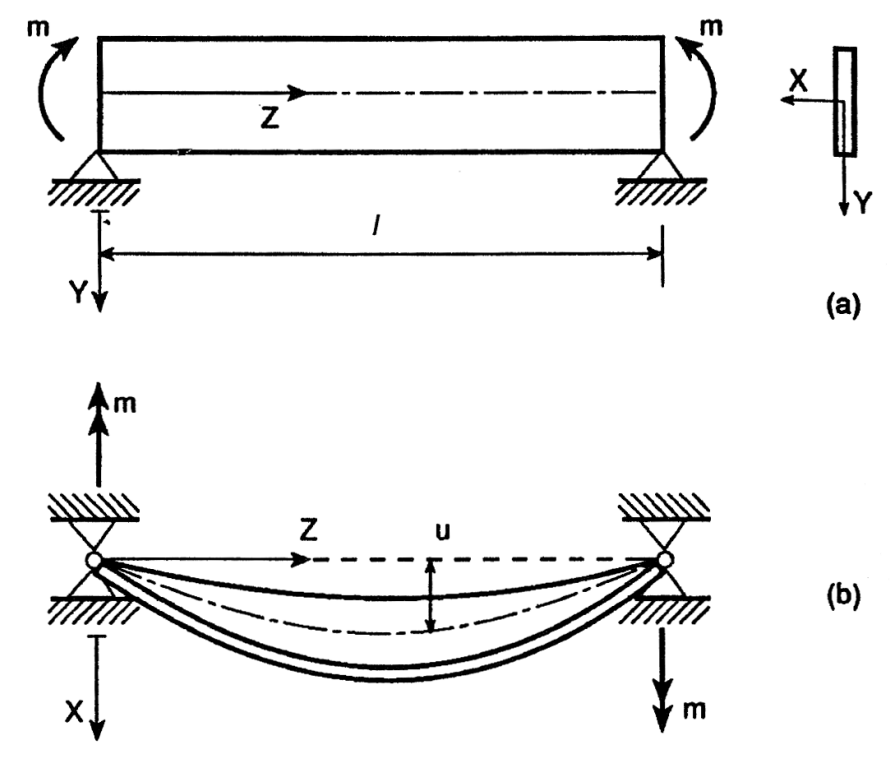

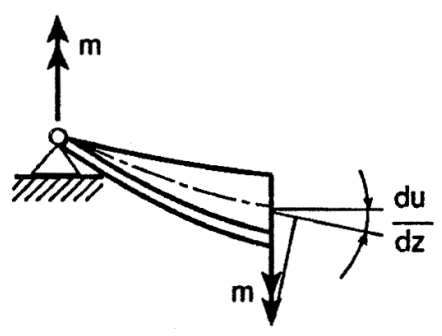

(c)

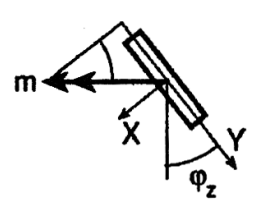

(d)

Figure 10. Scheme of a beam subjected to lateral-torsional buckling.

A solution to the system (47) can be found in the following variable-separable form [25]:

$$
\begin{aligned}
u(z, t) & =U(t) \eta(z), \\
\varphi_{z}(z, t) & =\Phi(t) \psi(z),
\end{aligned}
$$

where the functions $\eta(z)$ and $\psi(z)$ are such that the boundary conditions $\eta(0)=\eta(l)=\eta^{\prime \prime}(0)=\eta^{\prime \prime}(l)=\psi(0)=\psi(l)=0$ are satisfied. According to Bolotin [25], we can assume $\eta(z)=\psi(z)=\sin \frac{n \pi z}{l}$, with $n$ being a natural number. In this case, we obtain the following matrix form:

$$
\left[\begin{array}{cc}
\mu & 0 \\
0 & \mu \rho^{2}
\end{array}\right]\left\{\begin{array}{l}
\ddot{U} \\
\ddot{\Phi}
\end{array}\right\}+\left[\begin{array}{cc}
E I_{y} \frac{n^{4} \pi^{4}}{l^{4}} & 0 \\
0 & G I_{t} \frac{n^{2} \pi^{2}}{l^{2}}
\end{array}\right]\left\{\begin{array}{l}
U \\
\Phi
\end{array}\right\}-m\left[\begin{array}{cc}
0 & \frac{n^{2} \pi^{2}}{l^{2}} \\
\frac{n^{2} \pi^{2}}{l^{2}} & 0
\end{array}\right]\left\{\begin{array}{l}
U \\
\Phi
\end{array}\right\}=\left\{\begin{array}{l}
0 \\
0
\end{array}\right\},
$$


which can be symbolically rewritten as:

$$
[M]\{\ddot{q}\}+[K]\{q\}-m\left[K_{g}\right]\{q\}=\{0\},
$$

where $\{q\}=(U, \Phi)^{\top}$. The mass matrix, $[M]$, the elastic stiffness matrix $[K]$, and the geometric stiffness matrix $\left[K_{g}\right]$ in Eq.(50) can be defined in comparison with Eq.(49). Looking for a general solution in the form $\{q\}=\left\{q_{0}\right\} \mathrm{e}^{\mathrm{i} \omega t}$, we obtain:

$$
\left([K]-m\left[K_{g}\right]-\omega^{2}[M]\right)\left\{q_{0}\right\}=\{0\}
$$

A nontrivial solution to Eq.(51) exists if and only if the determinant of the resultant coefficient matrix of the vector $\left\{q_{0}\right\}$ vanishes. This yields the following generalized eigenvalue problem:

$$
\operatorname{det}\left([K]-m\left[K_{g}\right]-\omega^{2}[M]\right)=0,
$$

where $m$ and $\omega^{2}$ are the eigenvalues of the system.

As limit cases, if $\mu=0$, then we obtain the critical bending moments given by the Prandtl's formula:

$$
m_{n c}=\frac{n \pi}{l} \sqrt{E I_{y} G I_{t}}
$$

whereas, if $m=0$, then we obtain the natural flexural and torsional eigenfrequencies of the beam:

$$
\begin{aligned}
\omega_{n}^{\text {flex }} & =\left(\frac{n \pi}{l}\right)^{2} \sqrt{\frac{E I_{y}}{\mu}}, \\
\omega_{n}^{\text {tors }} & =\frac{n \pi}{\rho l} \sqrt{\frac{G I_{t}}{\mu}} .
\end{aligned}
$$

Considering a rectangular beam with a depth to span ratio of $1 / 3$ and with a thickness to depth ratio of $1 / 10$, the evolution of the first two flexural and torsional eigenfrequencies of the system are shown in Fig.11 as functions of the applied bending moment. In this case, the curves in the nondimensional plane $\left(\omega / \omega_{1}^{\text {flex }}\right)^{2}$ vs. $m / m_{1 c}$ are no longer straight lines. This fact can be ascribed to the coupling between torsional and flexural vibrations of the beam. Moreover, when $m$ is increased from zero (pure resonance instability) up to the critical bending moment computed according to the Parandtl's formula (pure buckling instability), $m_{1 c}$, we note that the resonance frequencies related to flexural oscillations progressively decrease from $\omega_{1}^{\text {flex }}$ down to zero in correspondence of the critical bending moments given by the Prandtl's formula. Conversely, the resonance frequencies related to torsional oscillations increase. From the mathematical point of view, this is the result of the fact that the sum of the 


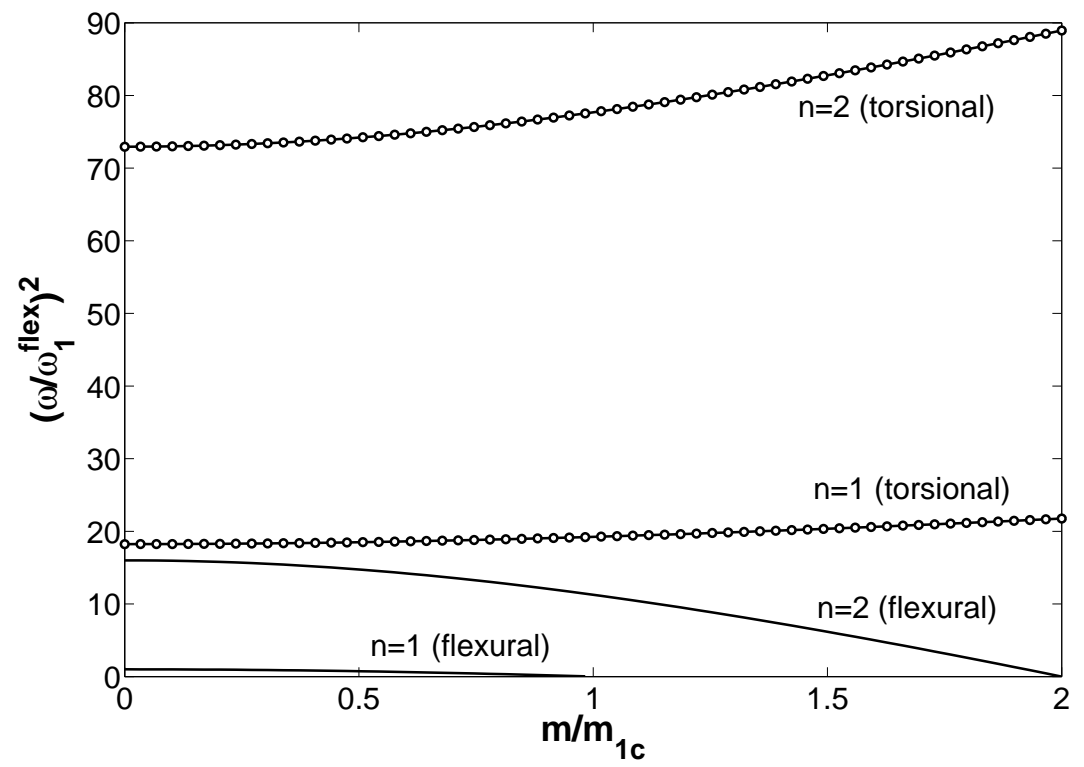

Figure 11. Nondimensional flexural and torsional frequencies vs. nondimensional bending moments for the continuous system in Fig.10.

squares of the two eigenfrequencies for a given value of $n$ is constant when the applied moment $m$ is varied.

\section{Finite elements procedure}

When the mechanical system cannot be reduced to the schemes previously analyzed, it is possible to apply the Finite Element Method [26,27]. According to this approach, the equations of motion for an elastic system with a finite number of degrees of freedom can be expressed in matrix form, taking also into account the effect of the geometric nonlinearity through the geometric stiffness matrix $[28,29]$.

For the sake of generality, let the elastic domain $V$ be divided into subdomains $V_{e}$, and let each element contain $m$ nodal points, each one having $g$ degrees of freedom. In compact form, the displacement vector field defined on the element $V_{e}$ may be represented as:

$$
\underset{(g \times 1)}{\left\{\eta_{e}\right\}}=\underset{g \times(g \times m)}{\left[\eta_{e}\right]} \underset{(g \times m) \times 1}{\left\{\delta_{e}\right\}}
$$

where $\left[\eta_{e}\right]$ is the matrix collecting the shape functions and $\left\{\delta_{e}\right\}$ is the nodal displacements vector. 
The deformation characteristic vector is obtained by derivation:

$$
\underset{(d \times 1)}{\left\{q_{e}\right\}}=\underset{(d \times g)}{\left[\begin{array}{l}
(g \times 1) \\
\left(\eta_{e}\right\}
\end{array},\right.}
$$

where $[\partial]$ is the kinematic operator relating strains to displacements, whereas $d$ denotes the dimension of the strain characteristic vector, e.g. $d=3$ for a beam in plane or $d=6$ for a beam in space. Hence, introducing Eq.(55) into Eq.(56), we obtain:

$$
\underset{(d \times 1)}{\left\{q_{e}\right\}}=\underset{(d \times g)}{[\partial]} \underset{g \times(g \times m)}{\left[\eta_{e}\right]} \underset{(g \times m) \times 1}{\left\{\delta_{e}\right\}}=\underset{d \times(g \times m)}{\left[B_{e}\right]} \underset{(g \times m) \times 1}{\left\{\delta_{e}\right\}},
$$

where the matrix $\left[B_{e}\right]$ is calculated by derivation of the shape functions.

According to these definitions, we can obtain the following matrix equation for each finite element (see [1], Ch.11 for more details):

$$
\left[M_{e}\right]\left\{\ddot{\delta_{e}}\right\}+\left(\left[K_{e}\right]-\left[K_{g e}\right]\right)\left\{\delta_{e}\right\}=\{0\},
$$

where $\left[M_{e}\right],\left[K_{e}\right]$ and $\left[K_{g e}\right]$ denote, respectively, the local mass matrix, the local elastic stiffness matrix and the local geometric stiffness matrix of the finite element.

As usual, the local mass and elastic stiffness matrices are given by [26, 27]:

$$
\begin{aligned}
{\left[M_{e}\right] } & =\int_{V_{e}}\left[\eta_{e}\right]^{\top}[\mu]\left[\eta_{e}\right] \mathrm{d} V, \\
{\left[K_{e}\right] } & =\int_{V_{e}}\left[B_{e}\right]^{\top}[H]\left[B_{e}\right] \mathrm{d} V .
\end{aligned}
$$

The local geometric stiffness matrix can be computed as follows [28]:

$$
\left[K_{g e}\right]=\int_{V_{e}}\left[G_{e}\right]^{\top}\left[S_{e}\right]\left[G_{e}\right] \mathrm{d} V
$$

where the matrix $\left[S_{e}\right]$ is related to the components of the stress field:

$$
\left[S_{e}\right]=\left[\begin{array}{ccc}
\sigma_{x}\left[I_{3}\right] & \tau_{x y}\left[I_{3}\right] & \tau_{x z}\left[I_{3}\right] \\
\tau_{x y}\left[I_{3}\right] & \sigma_{y}\left[I_{3}\right] & \tau_{y z}\left[I_{3}\right] \\
\tau_{x z}\left[I_{3}\right] & \tau_{y z}\left[I_{3}\right] & \sigma_{z}\left[I_{3}\right]
\end{array}\right]
$$

where $\left[I_{3}\right]$ denotes a unit matrix with dimensions $(3 \times 3)$. The matrix $\left[G_{e}\right]$ is related to the first derivative of the shape functions through the differential operator $[\widehat{\partial}]$ :

$$
\underset{(g \times g) \times(g \times m)}{\left[G_{e}\right]}=\underset{(g \times g) \times g}{[\widehat{\partial}]} \underset{g \times(g \times m)}{\left[\eta_{e}\right]},
$$


where

$$
[\widehat{\partial}]=\left[\begin{array}{l}
\frac{\partial}{\partial x}\left[I_{3}\right] \\
\frac{\partial}{\partial y}\left[I_{3}\right] \\
\frac{\partial}{\partial z}\left[I_{3}\right]
\end{array}\right]
$$

According to this formulation, we note that the geometric stiffness matrix is a function of the stress components through the matrix $\left[S_{e}\right]$. In the case of a compressive stress field, the geometric stiffness terms become negative and reduce the corresponding elements of the local elastic stiffness matrix, just as shown for the discrete mechanical systems. We also remark that this formulation is quite general, since the information related to the finite element topology is simply included in the matrix $\left[\eta_{e}\right]$ which collects the shape functions and in the differential operator $[\partial]$ (see [1] for more details).

By performing the usual operations of rotation, expansion and assemblage of the mass, elastic stiffness and geometric stiffness matrices of the element, Eq.(58) can be written in global form:

$$
[M]\{\ddot{\delta}\}+\left([K]-\lambda\left[K_{g}\right]\right)\{\delta\}=\{0\}
$$

Looking for the solution to Eq.(64) in the general form $\{\delta\}=\left\{\delta_{0}\right\} \mathrm{e}^{\mathrm{i} \omega t}$, where $\omega$ is the natural frequency of the system, we can formulate the generalized eigenproblem as in the cases discussed above:

$$
\operatorname{det}\left(\left[K_{e}\right]-\lambda\left[K_{g}\right]-\omega^{2}\left[M_{e}\right]\right)=0
$$

Therefore, the numerical procedure for the determination of the frequencyloading multiplier diagram consists in the following steps.

(1) For a given loading configuration defined by the loading multiplier $\lambda$, determine the stress field according to a linear elastic stress analysis.

(2) Compute the local mass matrix, the local elastic stiffness matrix and the local geometric stiffness matrix for each finite element.

(3) Perform the rotation, expansion and assemblage operations to obtain the global matrices.

(4) Solve the generalized eigenvalue problem of Eq.(65) and find the eigenfrequencies of the system, $\omega_{i}^{2}$, with $i=1, \ldots, g \times n$.

(5) Iterate the above-described procedure for different values of $\lambda$. 


\section{Discussion and conclusions}

The problems of elastic instability (buckling) and dynamic instability (resonance) have been the subject of extensive investigation and have received a large attention from the structural mechanics community. Nonetheless, the study of the interaction between these elementary forms of instability is still an open point.

The phenomenon of flutter instability of the Tacoma Narrows Bridge occurred on November 7, 1940, can be reinterpreted as the result of such a catastrophic interaction. This cable-suspended bridge was solidly built, with girders of carbon steel anchored in huge blocks of concrete and was the first of its type to employ plate girders to support the roadbed. While in the earlier designs any wind would simply pass through the truss, in the new design of the 1940 the wind would be diverted above and below the structure. Shortly after construction, it was discovered that the bridge would sway and buckle dangerously in windy conditions. This resonance was flexural, meaning the bridge buckled along its length, with the roadbed alternately raised and depressed in certain locations. However, the failure of the bridge occurred when a never-before-seen twisting mode occurred.

A Report to the Federal Works Agency [30] excluded the phenomenon of pure forced resonance as the actual reason of instability: "...it is very improbable that resonance with alternating vortices plays an important role in the oscillations of suspension bridges. First, it was found that there is no sharp correlation between wind velocity and oscillation frequency such as is required in case of resonance with vortices whose frequency depends on the wind velocity...". A new theory for the interpretation of these complex aerodynamic instabilities was developed by Scanlan [21,22] and then elaborated by various researchers [31-34]. Basically, the so-called flutter theory considers the following equation of motion for the mechanical system in the finite element framework $[9,32]$ :

$$
[M]\{\ddot{\delta}\}+[C]\{\dot{\delta}\}+[K]\{\delta\}=\{F\}_{m i}+\{F\}_{m d}
$$

where $\{F\}_{m i}$ and $\{F\}_{m d}$ are, respectively, the motion-independent wind force vector and the motion-dependent aeroelastic force vector. A special attention is given to the structural damping, which is included in the equations of motion through the damping matrix $[C]$. The motion-dependent force vector is then put in relationship with the nodal displacements of the system, $\{\delta\}$, and the nodal velocities, $\{\dot{\delta}\}$, according to the flutter derivative matrices, $\left[K^{*}\right]$ and $\left[C^{*}\right]$, that are empirically determined in the wind tunnel by using section models of the bridge. As a result, the problem becomes highly nonlinear, and the flutter velocity, $U_{c r}$, and the flutter frequency, $\omega_{c r}$, can be determined from 
the following eigenproblem:

$$
\operatorname{det}\left([K]-\frac{1}{2} \rho U_{c r}^{2}\left[K^{*}\right]-\omega_{c r}^{2}[M]+\omega_{c r}[C]-\frac{1}{2} \rho U_{c r} \omega_{c r}\left[C^{*}\right]\right)=0,
$$

where $\rho$ is the air density and $1 / 2 \rho U_{c r}^{2}$ is the wind pressure.

It is important to remark that this eigenproblem shares most of the features of the generalized eigenproblem that we have analyzed in the present study. In fact, in both cases, two eigenvalues have to be determined from the eigenequation. However, the flutter theory gives prominence to the role played by the structural damping, although being generally less than $1 \%$ (see e.g. [32]). Moreover, the value of the mechanical damping seems to represent a sort of free parameter in the model. In fact, this parameter is usually assumed, like in [32], rather than experimentally evaluated. Another difference with our proposed approach relies in the geometric stiffness matrix, which is not taken into account in the current flutter theory. However, due to the large structural displacements, the flutter derivative matrix $\left[K^{*}\right]$ plays a very similar role, although being experimentally obtained, rather than analytically computed.

In conclusion, it seems to be possible to reinterpret the phenomenon of aeroelastic instability as the result of the interaction between pure resonance and pure buckling instabilities. According to our approach, the geometric stiffness matrix has a preeminent role and the mechanical damping can be neglected, as usually done in most of the structural engineering applications. On this line, the collapse of the Tacoma Narrows bridge can be considered as the result of the interaction between buckling (related to the wind pressure proportional to the square of the wind velocity) and resonance (caused by the frequency of the wind gusts). Thus, this would give a new explanation on why the Tacoma Narrows bridge failure took place under moderate wind velocities (wind pressure lower than the critical buckling load) and wind gusts frequencies different from the natural frequencies of the bridge. Future developments of the present work will regard the assessment of the proposed approach to the analysis of bridge instabilities, as well as the comparison with the classical flutter theory on the basis of real case histories.

\section{Appendix: orthonormality of the eigenfunctions of deflected beams subjected to an axial force}

As is well-known, the eigenfunctions $\eta_{i}$ of deflected beams computed according to pure modal analysis are orthonormal functions. It is possible to demonstrate that this fundamental property still holds when the beam is subjected to an axial load, $N$, as that shown in Fig.8. We may in fact write Eq.(38b) for two 
different eigensolutions:

$$
\begin{aligned}
\eta_{j}^{I V}+\beta^{2} \eta_{j}^{I I} & =\alpha_{j}^{4} \eta_{j}, \\
\eta_{k}^{I V}+\beta^{2} \eta_{k}^{I I} & =\alpha_{k}^{4} \eta_{k} .
\end{aligned}
$$

Multiplying Eq.(68a) by $\eta_{k}$ and Eq.(68b) by $\eta_{j}$, and integrating over the beam length, we obtain:

$$
\begin{aligned}
& \int_{0}^{l} \eta_{k} \eta_{j}^{I V} \mathrm{~d} z+\beta^{2} \int_{0}^{l} \eta_{k} \eta_{j}^{I I} \mathrm{~d} z=\alpha_{j}^{4} \int_{0}^{l} \eta_{k} \eta_{j} \mathrm{~d} z \\
& \int_{0}^{l} \eta_{j} \eta_{k}^{I V} \mathrm{~d} z+\beta^{2} \int_{0}^{l} \eta_{j} \eta_{k}^{I I} \mathrm{~d} z=\alpha_{k}^{4} \int_{0}^{l} \eta_{j} \eta_{k} \mathrm{~d} z .
\end{aligned}
$$

Integrating by parts the left-hand sides, the foregoing equations transform as follows:

$$
\begin{aligned}
& {\left[\eta_{k} \eta_{j}^{I I I}\right]_{0}^{l}-\left[\eta_{k}^{I} \eta_{j}^{I I}\right]_{0}^{l}+\int_{0}^{l} \eta_{k}^{I I} \eta_{j}^{I I} \mathrm{~d} z+\beta^{2}\left[\eta_{k} \eta_{j}\right]_{0}^{l}} \\
& -\beta^{2} \int_{0}^{l} \eta_{k}^{I} \eta_{j}^{I} \mathrm{~d} z=\alpha_{j}^{4} \int_{0}^{l} \eta_{k} \eta_{j} \mathrm{~d} z, \\
& {\left[\eta_{j} \eta_{k}^{I I I}\right]_{0}^{l}-\left[\eta_{j}^{I} \eta_{k}^{I I}\right]_{0}^{l}+\int_{0}^{l} \eta_{j}^{I I} \eta_{k}^{I I} \mathrm{~d} z+\beta^{2}\left[\eta_{j} \eta_{k}\right]_{0}^{l}} \\
& -\beta^{2} \int_{0}^{l} \eta_{j}^{I} \eta_{k}^{I} \mathrm{~d} z=\alpha_{k}^{4} \int_{0}^{l} \eta_{j} \eta_{k} \mathrm{~d} z .
\end{aligned}
$$

When each of the two ends of the beam is constrained by a built-in support $\left(\eta=\eta^{I}=0\right)$, or by a hinge $\left(\eta=\eta^{I I}=0\right)$, the quantities in square brackets vanish. On the other hand, when the end in $z=0$ is either unconstrained $\left(\eta^{I I I}=\eta^{I I}=0\right)$, or constrained by a double rod $\left(\eta^{I I I}=\eta^{I}=0\right)$, the remaining end of the beam has to be constrained either by a built-in support $\left(\eta=\eta^{I}=\right.$ $0)$, or by a simple support $\left(\eta=\eta^{I I}=0\right)$. For both configurations, only the terms $\left[\eta_{i} \eta_{k}\right]_{0}^{l}$ are different from zero.

In any case, subtracting member by member, these quantities are canceled and we have:

$$
\left(\alpha_{j}^{4}-\alpha_{k}^{4}\right) \int_{0}^{l} \eta_{j} \eta_{k}=0
$$

which leads to the orthonormality condition:

$$
\int_{0}^{l} \eta_{j} \eta_{k}=\delta_{i j}
$$

where $\delta_{i j}$ is the Kronecker delta. Thus, when the eigenvalues are distinct, the integral of the product of the corresponding eigenfunctions vanishes. When, instead, the indices $j$ and $k$ coincide, the condition of normality reminds us that the eigenfunctions are defined neglecting a factor of proportionality. 


\section{Acknowledgements}

The financial support provided by the European Union to the Leonardo da Vinci project "Innovative Learning and Training on Fracture (ILTOF)" is gratefully acknowledged.

\section{References}

[1] A. Carpinteri. Structural Mechanics: A Unified Approach. Chapman \& Hall, London, 1997.

[2] R.W. Clough and J. Penzien. Dynamics of Structures. McGraw-Hill, New York, 1975 .

[3] A. Carpinteri and N. Pugno. Towards chaos in vibrating damaged structures Part I: Theory and period doubling. Journal of Applied Mechanics, 72:511-518, 2005.

[4] A. Carpinteri and N. Pugno. Towards chaos in vibrating damaged structures Part II: Parametrical investigation. Journal of Applied Mechanics, 72:519-526, 2005 .

[5] H. Kawai. Bending and torsional vibration of tall buildings in strong wind. Journal of Wind Engineering and Industrial Aerodynamics, 50:281-288, 1993.

[6] M.A. Astiz. Flutter stability of very long suspension bridges. ASCE Journal of Bridge Engineering, 3:132-139, 1998.

[7] R. Scott. In the Wake of Tacoma: Suspension Bridges and the Quest for Aerodynamic Stability. ASCE, Reston, 2001.

[8] R.H. Scanlan. Aerodynamics of cable-supported bridges. Journal of Construction Steel Resources, 39:51-68, 1996.

[9] U. Starossek. Prediction of bridge flutter through the use of finite elements. Structural Engineering Review, 5:301-307, 1993.

[10] E.H. Simiu and R.H. Scanlan. Wind Effects on Structures. Wiley, New York, 1986.

[11] K.Y. Billah and R.H. Scanlan. Resonance, Tacoma Narrows bridge failure, and undergraduate physics textbooks. American Journal of Physics, 59:118-124, 1991.

[12] R.H. Scanlan. Toward introduction of empiricism in the wind design of longspan bridges. In Proceedings of the International Conference on Cable Stayed and Suspension Bridges, pages 15-28, 1994.

[13] A.M. Liapunov. General Problem of Stability of Motion. Kharkov (reproduced in Ann. Math. Studies 17, Princeton Univ. Press, Princepton 1949), 1892. 
[14] V.V. Bolotin. Non Conservative Problems of the Theory of Elastic Stability. Pergamon Press, New York, 1963.

[15] V.V. Bolotin. The Dynamic Stability of Elastic Systems. Holden-Day, San Francisco (Russian original: Moscow, 1956), 1964.

[16] A.P. Seyranian. Stability and Catastrophes of Vibrating Systems Depending on Parameters. Technical University of Denmark, Lyngby, Denmark, 1991.

[17] A.P. Seyranian and A.A. Mailybaev. Multiparameter Stability Theory with Mechanical Aplications. World Scientific Publishing Co. Pte. Ltd., 2003.

[18] K. Huseyin. Multiple Parameter Stability Theory and its Applications: Bifurcation, Catastrophes, Instabilities. Clarendon, Oxford, 1986.

[19] K. Huseyin. Dynamics, tability, and bifurcations. ASME Applied Mechanics Reviews, 55:R5-R15, 2002.

[20] Z. Elfelsoufi and L. Azrar. Buckling, lutter and vibration analyses of beams by integral equation formulations. Computers \& Structures, 83:2632-2649, 2005.

[21] R.H. Scanlan and J.J. Tomko. Airfoil and bridge deck flutter derivatives. Journal of Engineering Mechanics, 97:1717-1737, 1971.

[22] R.H. Scanlan. The action of flexible bridges under winds, I: flutter theory. Journal of Sound and Vibration, 60:187-199, 1978.

[23] D.H. Hodges and G.A. Pierce. Introduction to Structural Dynamics and Aeroelasticity. Cambridge University Press, Cambridge, UK, 2002.

[24] R.H. Scanlan. State-of-the-art methods for calculating flutter, vortex-induced and buffeting response of bridge structures. Federal Highway Administration, Report No. FHWA/RD-80/050, Washington, DC, 1981.

[25] V.V. Bolotin. Dynamic stability of structures. In A.N. Kounadis and W.B. Kratzig, editors, Nonlinear Stability of Structures: Theory and Computational Techniques, pages 3-72. Springer, Wien, 1995.

[26] K.J. Bathe. Finite Element Procedures in Engineering Analysis. Prentice-Hall Inc., Englewood Cliff, N.J., 1982.

[27] O.C. Zienkiewicz and R.L. Taylor. The Finite Element Method for Solid and Structural Mechanics. Elsevier, 6th Edition, Amsterdam, 2005.

[28] A. Rutenberg. Simplified P-Delta analysis for asymmetric structures. ASCE Journal of Structural Division, 108:1995-2013, 1982.

[29] J.S. Przemieniecki. Theory of Matrix Structural Analysis. Dover, New York, 1985.

[30] O.H. Ammann, T. Von Karman, and G.B. Woodruff. The failure of the Tacoma Narrows bridge. Report to the Federal Works Agency, March 28, 1941. 
[31] M. Como, A. Grimaldi, and F. Maceri. Statical behaviour of long-span cablestayed bridges. International Journal of Solids and Structures, 21:831-850, 1985 .

[32] A. Namini, P. Albrecht, and H. Bosch. Finite element-based flutter analysis of cable-suspended bridges. ASCE Journal of Structural Engineering, 118:15091526, 1992.

[33] F. Brancaleoni and G. Diana. The aerodynamic design of the Messina Straits bridge. Journal of Wind Engineering and Industrial Aerodynamics, 48:395-409, 1993.

[34] F. Brancaleoni and D.M. Brotton. The role of time integration in suspension bridge dynamics. International Journal for Numerical Methods in Engineering, 20:715-732, 2005. 